Z. Wahrscheinlichkeitstheorie verw. Gebiete 61, $291(1982)$
Zeitschrift für

Wahrscheinlichkeitstheorie

und verwandte Gebiete

(C) Springer-Verlag 1982

\title{
Correction to \\ On Weak Convergence to Brownian Motion
}

Z. Wahrscheinlichkeitstheorie verw. Gebiete 52, 251-265 (1980)

Inge S. Helland

Department of Mathematics and Statistics,

Agricultural University of Norway, 1432 Aas-NLH, Norway

Adam Jakubowski and Leszek Słomiński have pointed out an error in my proof of Proposition (4.5). The statement that " $V(x, S)$ cannot increase when $S$ is refined" (the top of p. 261) is not true. One way of repairing the proof, is to define

$$
V_{1}(x, S)=\sum_{k=1}^{p} \int_{s_{k-1}}^{s_{k}} \sup _{s_{k-1} \leqq u<s_{k}}|x(s)-x(u)| d s .
$$

Then the proof can be carried through more or less as it stands with $V(x, S)$ replaced by $V_{1}(x, S)$. Since $V(x, S) \leqq V_{1}(x, S)$, the assertion containing (4.7) will continue to be true.

Received April 26, 1982 